\title{
Supersymmetry and Unification: Heavy Top Was the Key
}

\author{
Goran Senjanović \\ ICTP, Trieste, Italy
}

\begin{abstract}
I review the unification of gauge couplings of strong, weak and electro-magnetic interactions. I start by recalling the history of the most important prediction of low-energy supersymmetry: the correct value of the weak mixing angle tied to a large top quark mass. I then turn to the discussion of the present day situation of the minimal supersymmetric Grand Unified Theories based on $S U(5)$ and $S O(10)$ groups, and I show why the minimal $S U(5)$ is in accord with experiment. For the sake of completeness I also summarize the problems and possible solutions of the minimal ordinary $S U(5)$. One version, based on the minimal Georgi-Glashow model, augmented by the adjoint fermion, predicts a light fermion triplet to lie below $\mathrm{TeV}$ or so. Its (lepton number violating) decays offer a hope of probing neutrino (Majorana) masses and mixings at the LHC.
\end{abstract}

\section{Contents}

\section{Foreword}

2 Unification of gauge couplings: low energy supersymmetry and heavy top

\section{Minimal SU(5)}

\section{Minimal supersymmetric SU(5)}

$5 \mathrm{SO}(10)$

\section{Conclusions}

\section{Outlook}

\section{Foreword}

There can be no pleasure contributing to a memorial volume, especially if it is dedicated to a great physicist and an outstanding human being. It is a great honor though. I got to know Julius Wess some thirty years ago, and he was a good friend. He was also a great friend of Balkan physics, especially what once upon a time was called Yugoslav physics. The passage of time naturally brings nostalgia, and as I am writing this I cannot help but feel nostalgia for the golden days of our spring when we met for the first time. We both lectured in the 1983

\footnotetext{
${ }^{\S}$ To be published in the proceedings of the Scientific and Human Legacy of Julius Wess, Memorial Workshop held in Donji Milanovac, Serbia, August 2011. There is plagiarism in my title. It is borrowed from the beautiful historical overview of the $\mathrm{V}$-A theory by Weinberg 1 .
}

Adriatic School in Dubrovnik, an excellent event organized jointly by the Belgrade and Zagreb High Energy groups. Julius was immediately hooked to the beauty of Dubrovnik, and fell in love with the atmosphere of hospitality and friendliness which he associated with the old Yugoslavia. He kept coming back regularly to both Croatia and Serbia and it is more than appropriate that his memorial workshop was held in the Djerdap National Park, one of the jewels of Serbia.

Julius Wess, as everybody knows, was one of the fathers of supersymmetry, which for the last four decades has been shaping our field, for better or for worse. I was introduced to supersymmetry in 1974, just as I was starting my $\mathrm{Ph}$. D. research, when I heard a talk by Bruno Zumino at Columbia University. He and Julius had just finished their classic work, and Zumino gave an inspiring talk. I for one was enchanted by the beauty of supersymmetry, although I could share Zumino's sentiment when he compared supersymmetry to a giraffe, saying: "I am sure we all agree that a giraffe is truly beautiful, but she doesn't seem to serve any purpose". As much as he was right about everything else in his talk, in this he was wrong: giraffes, as all living creatures, have an important role in preserving the ecological balance of nature. It is ironic that over the years supersymmetry would become the main candidate for the theory beyond the Standard Model. If anything, it found probably too much of a practical role, and today is not even considered "exotics" at the LHC. But then, it is neither first nor the last time that heresy becomes dogma. 


\section{Unification of gauge couplings: low energy supersymmetry and heavy top}

Around the same time, in 1974, Grand Unification started to emerge as a natural next step of the Standard Model unification, and brought in its huge scale of at least $10^{15} \mathrm{GeV}$ as dictated by proton stability. This made the so-called hierarchy question of the smallness of the electroweak scale compared to the Planck scale much more acute, for Grand Unification also brought in extreme finetuning, at least in its minimal versions. In the next five years or so, the Standard Model was to emerge triumphantly as the theory of both the charged and neutral weak processes. The theory agreed with all the data for the value of the weak mixing angle $\sin ^{2} \theta_{W}\left(M_{W}\right) \simeq 0.21$. This fit perfectly with the minimal $S U(5)$ theory of Georgi and Glashow [2], after Georgi, Quinn and Weinberg[3] taught us how to compute $\sin ^{2} \theta_{W}\left(M_{W}\right)$. They also taught us how to compute the unification scale and thus the proton lifetime, around $10^{30} \mathrm{yr}$ or so. As Maurice Goldhaber put it nicely, many experimentalists then rushed underground all across the globe, to look for proton decay.

All looked well, not a cloud on the horizon, except for the growing uneasiness about the hierarchy problem. And then it became clear that low energy supersymmetry around $\mathrm{TeV}$ or so (notice or so, we will come back to this later) would, if not cure the hierarchy problem, alleviate it greatly for you could do your fine-tuning at the tree level and live happily ever after.

However, it was soon pointed out by Dimopoulos, Raby and Wilczeck[4] (and, we will learn later, independently by Ibáñez and Ross [5]) that that the scale of unification and predicted proton lifetime get significantly increased in the minimal supersymmetric GUTs, while $\sin ^{2} \theta_{W}\left(M_{W}\right)$ remains almost unaffected.

Inspired by Dimopoulos et.al., in the fall of 1981, Bill Marciano and I decided to do a careful twoloop analysis of the unification with low energy supersymmetry, [6] and found out that Einhorn and Jones were doing it already (their paper [7] appropriately appeared before ours). The result was an increased $\sin ^{2} \theta_{W}\left(M_{W}\right) \simeq 0.23$, in clear disagreement with claimed value $\sin ^{2} \theta_{W}\left(M_{W}\right)_{\exp } \simeq 0.21$. Bill and I liked the idea of low energy supersymmetry and decided to stick our necks out in its favor. First of all, if you took into account the $\rho$ parameter of the Standard Model, the fit was actually

$$
\sin ^{2} \theta_{W}\left(M_{W}\right) \simeq 0.22-0.49\left(1-\rho^{2}\right) .
$$

In the Standard Model, with radiative corrections included, the (wrongly) quoted value was $\rho \simeq 0.98$, assuming that the top quark was going to be light according to the prejudice of the day. However, with a heavy top quark, around $200 \mathrm{GeV}$ or so, you would get $\rho \simeq 1.01$, and $\sin ^{2} \theta_{W}\left(M_{W}\right)_{\exp } \simeq 0.23$. Bill and I then argued in favor of low energy supersymmetry and a heavy top, and thus predicted the correct value of $\sin ^{2} \theta_{W}\left(M_{W}\right)$ ten years before LEP was to confirm it. Moreover, the top, lo and behold, turned out to be heavy. Looking back, I feel both proud and happy that we had the courage and sense to say it in print.

Historia magistra vitae, says the Latin proverb: history is the teacher of life. I don't know what this little history teaches us, but I think it was appropriate to bring it up. After all, it is not well known, not enough, that the successful unification, the greatest success of low energy supersymmetry, went hand in hand with the heavy top quark, long before experiment.

In what follows, I discuss the implementation of gauge coupling unification with grand unified theories. I argue that the $\mathrm{TeV}$ scale of supersymmetry ought be taken as soft, and that there is no guarantee that supersymmetry lies at the LHC reach. There was too much optimism in arguing so, and a negative result should not be taken against this beautiful idea, but rather against false prophets.

\section{Minimal SU(5)}

It was the minimal $\mathrm{SU}(5)$ that caused the underground rush, for it predicted proton lifetime on the order of $\tau_{p} \simeq 10^{30} \mathrm{yr}$. And on top, it also predicted the nucleon decay branching ratios as shown by Mohapatra 8 . Unfortunately these predictions resulted from the wrong mass relations: $m_{e}=m_{d}$. These relations can be corrected easily by simply adding higher dimensional operators [9], but then the theory stops being predictive.

In any case this is only history now, for the theory is not consistent, since

- gauge couplings do not unify

- neutrinos stay massless (as in the MSM).

Possible higher dimensional operators are not enough: neutrino mass comes out too small. It is 
important to know then what the minimal consistent realistic extensions are. Two such models were constructed, by adding

- a symmetric complex scalar field [10 (and higher dimensional operators for charged fermion masses)

$15_{H}=\left(1_{C}, 3_{W}\right)+\left(6_{C}, 1_{W}\right)+\left(3_{C}, 2_{W}\right)$,

with $\left(1_{C}, 3_{W}\right)$ being the usual Higgs triplet behind the type II seesaw [11]. The leptoquarks $\left(3_{C}, 2_{W}\right)$ may remain light (but not necessarily) and offer an interesting phenomenology [12. There is also an important prediction, a fast proton decay on the edge of experimental limits.

- an adjoint fermion field[13]

$24_{F}=\left(8_{C}, 1_{W}\right)+\left(1_{C}, 3_{W}\right)+\left(1_{C}, 1_{W}\right)+\ldots$

The fields $S=\left(1_{C}, 1_{W}\right)$ and $T=\left(1_{C}, 3_{W}\right)$ are responsible for type I[14] + III[15] hybrid seesaw. Again, one needs higher dimensional operators both for charged femions and for realistic neutrino Dirac yukawa couplings

This model predicts a light fermion triplet $\mathrm{T}$, with a mass below $\mathrm{TeV}$ so that the running of the $\mathrm{SU}(2)$ gauge coupling is slowed down and meets $\mathrm{U}(1)$ above $10^{15} \mathrm{GeV}$. Its phenomenology is quite interesting for it leads to lepton number violation at colliders in the form of same sign di-leptons as suggested originally long ago [16. For the relevant studies in the context of the type III seesaw, see Ref. [17], and for this specific model, see Ref. [18].

The two theories have in common a "fast" proton decay, with $\tau_{p} \leq 10^{35} \mathrm{yr}$.

\section{Minimal supersymmetric SU(5)}

The underground rush continued, or better to say, got boosted with the success of the minimal supersymmetric SU(5). As discussed in the Introduction, low energy supersymmetry, suggested in order to stabilize the Higgs mass hierarchy predicted correctly $\sin ^{2} \theta_{W} \simeq 0.23$, ten years before its confirmation at LEP.

The unification scale too was predicted: $M_{G U T} \simeq$ $10^{16} \mathrm{GeV}$ and in turn $\tau_{p}(d=6) \simeq 10^{36 \pm 1} \mathrm{yr}$, which would have rendered proton decay out of reach of experiment. However, supersymmetry leads to new contribution: $d=5$ operators [19] through the exchange of heavy color triplet Higgsino ( $T$ and $\bar{T})$. A rough estimate gives

$$
G_{T} \simeq \frac{\alpha}{4 \pi} y_{u} y_{d} \frac{m_{\text {gaugino }}}{M_{T} m_{\tilde{f}}^{2}} \simeq 10^{-30} \mathrm{GeV}^{-2},
$$

which for $y_{u} \simeq y_{d} \simeq 10^{-4}, m_{\text {gaugino }} \simeq 100 \mathrm{GeV}$, $m_{\tilde{f}} \simeq \mathrm{TeV}$ and $M_{T} \simeq 10^{16} \mathrm{GeV}$ gives $\tau_{p}(d=5) \simeq$ $10^{30-31} \mathrm{yr}$. It would seem that today this theory is ruled out. It was actually proclaimed dead [20], but then caution was raised for two important reasons: i) the uncertainty in sfermion masses and mixings 21 and ii) uncertainty in $M_{T}$ 22] due to necessity of higher dimensional operators 9 to correct bad fermion mass relations $m_{d}=m_{\ell}$. The $d=4$ operators, besides correcting these relations also split the masses of $m_{3}$ and $m_{8}$ of the weak triplet and color octet, respectively, in the adjoint $24_{H}$ Higgs super multiplet. One gets

$$
\left.\begin{array}{l}
M_{G U T}=M_{G U T}^{0}\left(\frac{M_{G U T}^{0}}{\sqrt{m_{3} m_{8}}}\right)^{1 / 2} \\
M_{T}=M_{T}^{0}\left(\frac{m_{3}}{m_{8}}\right)^{5 / 2}
\end{array}\right\} M_{G U T}^{0} \simeq 10^{16} \mathrm{GeV}
$$

where $M_{G U T}^{0}$ denotes the GUT scale predicted for $m_{3}=m_{8}$ at the tree level with $\mathrm{d}=5$ operators neglected. The fact that $M_{G U T}$ goes up by lowering $m_{3,8}$ was noticed quite some time ago [23]. In principle, the ratio of the triplet and octet masses can be as large as one wishes, so at first glance the proton lifetime would seem not to be limited from above at all. However, in recent years, Dvali [24] has been arguing that the effective strong gravity scale is given by $\Lambda_{\mathrm{gr}}=M_{P l} / \sqrt{N}$, where $M_{P l} \simeq 10^{18}$ and $N$ is the number of states for a theory in question. With $N$ around 100, in order for this theory to be perturbative, one must demand $M_{\mathrm{GUT}} \lesssim 10^{17} \mathrm{GeV}$ or $\tau_{p} \lesssim 10^{35}$ yrs.

In short, the minimal supersymmetric $S U(5)$ is still a perfectly viable theory, and the $d=5$ proton decay is close to the present limit. This minimal theory must account for neutrino masses and mixings and the only possibility is through the violation of R-parity. The first important implication of not assuming R-parity is that the lightest neutralino cannot be dark matter for it decays too fast with the collider signature of lepton number violation. Thus the only dark matter candidate is an unstable gravitino [25], and it works perfectly in the MSSM, see [26]. 


\section{$5 \mathrm{SO}(10)$}

Although $S U(5)$ is the minimal theory of grand unification and as such deserves maximal attention as a laboratory for studying proton decay, $S O(10)$ has important merits

- it unifies a fermion family in a spinorial $16_{F}$ representation and as such is a minimal unified theory of matter and interactions,

- it automatically contains right-handed neutri$\operatorname{nos} N$,

- it gives naturally $M_{N} \gg M_{W}$ and so neutrino has a tiny mass through the see-saw mechanism,

- in supersymmetry R-parity is a gauge symmetry [27],

- in the renormalizable version R-parity remains exact 28] (as in any B-L symmetric theory [29]), implying the stable lightest superpartner, natural dark matter candidate.

All the fermion masses and mixings can be accounted for with the $10_{H}$ and $126_{H}$ representations, the latter providing a large mass to the righthanded neutrinos. While the non supersymmetric, $\mathrm{SO}(10)$ theory was studied at length over the years, no predictive realistic model of fermion masses and mixings ever emerged.

If $10_{H}$ is taken to be real, the predictions turn out to be wrong [30, and making it complex doubles the Yukawas and kills the predictions.

This situation improves in supersymmetry, since holomorphicity guarantees single Yukawas for both $10_{H}$ and $126_{H}$. The minimal supersymmetric version, coined renormalizable, suggested already in 1982 [33] and rediscussed ten years later [32], has been under scrutiny in recent years [31. An important boost was provided by an observation that $b-\tau$ unification, tied with the type II seesaw, gives naturally large atmospheric mixing angle [34]. Moreover, it predicts $\theta_{13} \simeq 10^{\circ}$ [35], in accord with T2K [36] and Daya Bay [37] any need for flavour symmetries

After a great initial success, when pinned down precisely, the theory ran into tension between fast proton and neutrino masses. It was revisited [38] and shown to work with the so-called split supersymmetry spectrum of heavy sfermions [39]. Although by splitting the supersymmetric spectrum one loses the hierarchy protection, the unification of gauge couplings is preserved. If one accepts split supersymmetry, one can also develop a realistic model [40] based on a beautiful radiative mechanism [41].

Instead of a large $126_{H}$ Higgs, one may choose a $16_{H}$ and then build $126_{H}$ Higgs effectively as $16_{H}^{2}$. This induces a proliferation of couplings and needs extra flavor symmetries, and thus goes beyond a simple GUT picture; see e.g. 42.

\section{Conclusions}

I gave here an account of the unification of gauge couplings in supersymmetry, and showed how it prophetically predicted a heavy top quark, some ten years before LEP and some fifteen years before the top discovery. This still remains the main prediction of low energy supersymmetry, giving us hope to discover it at the LHC. We should keep in mind though that it can easily be above the LHC reach, without losing unification, only at the expense of some additional fine-tuning. I, for one, can live with that.

I then discussed the minimal supersymmetric grand unified theories based on $\mathrm{SU}(5)$ and $\mathrm{SO}(10)$ gauge groups as a way of completing the program of unification. It is remarkable that the minimal supersymmetric $\mathrm{SU}(5)$ is still in accord with experiment. I showed, on the other hand, that the computation of the proton decay branching ratios can be achieved in the $\mathrm{SO}(10)$ theory with split supersymmetry. For the lovers of unification who do not worry about the hierarchy problem this is OK, for splitting the supersymmetry preserves the unification of gauge couplings. I was rather telegraphic here for the lack of space; a reader who wants to learn more about this beautiful subject can have a look at my recent comprehensive review [43].

\section{Outlook}

We all know how quickly a heresy can turn into a dogma, and I must admit that the more supersymmetry was becoming a dogma over the years, the less I felt my original enthusiasm. Moreover, it was and still is, hard not to have doubts in a theory whose main prediction went hand in hand with a a desert in energies all the way to the unreachable GUT scale. Now that, according to many, it is getting into trouble I start to feel my old love again, 
and wish to defend it. It is more than an appropriate to paraphrase Mark Twain here: any rumor about the death of low energy supersymmetry has been greatly exaggerated.

I put my money where my mouth is and in 2003 , at a conference held in Vrnjačka Banja, Serbia, I made a bet with Julius that supersymmetry would be discovered at the LHC. He bet against it, so that either way it was a win-win situation for him because he was going to gain much more by losing the bet (btw, it was a serious bet, a case of expensive wine). It may appear strange that I would bet in favor of supersymmetry in view of my doubts. But then again, I too have a vested interest in low energy supersymmetry, and either outcome would be a win for me as well. I only regret deeply that Julius will not be around to settle the bet.

Julius Wess will not only be missed by family, friends, colleagues and collaborators, but by many of us from the old Yugoslavia who recall with warmth his love and support for these countries. I for one will miss very much seeing him, as happened many times, in these lands so dear to me.

\section{Acknowledgments}

I am deeply grateful to the organizers of the Julius Wess Memorial Workshop for giving me the opportunity to honor a great physicist. It would take too much space to thank all of my collaborators on the topics discussed here, to whom I am deeply indebted. I wish though to acknowledge with great pleasure my original collaboration with Bill Marciano on the issue of supersymmetric unification. I thank Bill and Borut Bajc for careful reading of this manuscript and for useful comments. Special thanks are due to Alejandra Melfo and Miha Nemevšek for discussions and help with this write-up.

\section{References}

[1] S. Weinberg, "V-A was the key," J. Phys. Conf. Ser. 196, 012002 (2009).

[2] H. Georgi and S. L. Glashow, "Unity Of All Elementary Particle Forces," Phys. Rev. Lett. 32, 438 (1974).

[3] H. Georgi, H. R. Quinn and S. Weinberg, "Hierarchy of Interactions in Unified Gauge Theories," Phys. Rev. Lett. 33, 451 (1974).
[4] S. Dimopoulos, S. Raby and F. Wilczek, "Supersymmetry And The Scale Of Unification," Phys. Rev. D 241681 (1981).

[5] L.E. Ibáñez and G.G. Ross, "Low-Energy Predictions In Supersymmetric Grand Unified Theories," Phys. Lett. B 105, 439 (1981).

[6] W. Marciano and G. Senjanović, "Predictions Of Supersymmetric Grand Unified Theories," Phys. Rev. D 25, 3092 (1982).

[7] M.B. Einhorn and D.R. Jones, "The Weak Mixing Angle And Unification Mass In Supersymmetric SU(5)," Nucl. Phys. B 196, 475 (1982).

[8] R. N. Mohapatra, "Flavor Mixing In SU(5) Grand Unified Theories," Phys. Rev. Lett. 43, 893 (1979).

[9] J. R. Ellis and M. K. Gaillard, "Fermion Masses And Higgs Representations In SU(5)," Phys. Lett. B 88, 315 (1979).

[10] I. Doršner and P. Fileviez Pérez, "Unification without supersymmetry: Neutrino mass, proton decay and light leptoquarks," Nucl. Phys. B 723 (2005) 53 [hep-ph/0504276].

[11] M. Magg and C. Wetterich, "Neutrino Mass Problem And Gauge Hierarchy," Phys. Lett. B 94, 61 (1980).

R. N. Mohapatra and G. Senjanović, "Neutrino Masses And Mixings In Gauge Models With Spontaneous Parity Violation," Phys. Rev. D 23, 165 (1981).

G. Lazarides, Q. Shafi and C. Wetterich, "Proton Lifetime And Fermion Masses In An SO(10) Model," Nucl. Phys. B 181, 287 (1981).

[12] I. Doršner, S. Fajfer, J. F. Kamenik and N. Košnik, "Light colored scalars from grand unification and the forward-backward asymmetry in t t-bar production," Phys. Rev. D 81, 055009 (2010) [arXiv:0912.0972 [hep-ph]].

[13] B. Bajc and G. Senjanović, "Seesaw at LHC," JHEP 0708, 014 (2007) arXiv:hep-ph/0612029; B. Bajc, M. Nemevšek and G. Senjanović, "Probing seesaw at LHC," Phys. Rev. D 76, 055011 (2007) arXiv:hep-ph/0703080. 
[14] P. Minkowski, "Mu $\rightarrow$ E Gamma At A Rate Of One Out Of 1-Billion Muon Decays?," Phys. Lett. B 67, 421 (1977); R. Mohapatra and G. Senjanović, "Neutrino Mass And Spontaneous Parity Nonconservation," Phys. Rev. Lett. 44, 912 (1980); T. Yanagida, in Proc. Workshop on Unified Theories and Baryon Number in the Universe, eds. A. Sawada, A. Sugamoto, (KEK Report No. 79-18), Tsukuba 1979; S. Glashow, in Quarks and Leptons, Cargèse 1979, eds. M. Lévy. et al., (Plenum, 1980, New York); M. Gell-Mann, P. Ramond and R. Slansky, in Proc. Supergravity Stony Brook Workshop, New York, 1979, eds. P. Van Niewenhuizen, D. Freeman (NorthHolland, Amsterdam).

[15] R. Foot, H. Lew, X. G. He and G. C. Joshi, "Seesaw Neutrino Masses Induced by a Triplet of Leptons," Z. Phys. C 44, 441 (1989).

[16] W. Y. Keung and G. Senjanović, "Majorana Neutrinos And The Production Of The RightHanded Charged Gauge Phys. Rev. Lett. 50, 1427 (1983).

[17] R. Franceschini, T. Hambye and A. Strumia, "Type-III see-saw at LHC," Phys. Rev. D 78, 033002 (2008) [arXiv:0805.1613 [hep-ph]]; F. del Aguila and J. A. Aguilar-Saavedra, "Distinguishing seesaw models at LHC with multilepton signals," Nucl. Phys. B 813, 22 (2009) arXiv:0808.2468 [hep-ph]]. F. del Aguila and J. A. Aguilar-Saavedra, "Electroweak scale seesaw and heavy Dirac neutrino signals at LHC," Phys. Lett. B 672 (2009) 158 arXiv:0809.2096 [hep-ph]].

[18] A. Arhrib, B. Bajc, D. K. Ghosh, T. Han, G. Y. Huang, I. Puljak and G. Senjanović, "Collider Signatures for Heavy Lepton Triplet in Type I+III Seesaw," Phys. Rev. D 82, 053004 (2010) arXiv:0904.2390 [hep-ph]].

[19] N. Sakai and T. Yanagida, "Proton Decay In A Class Of Supersymmetric Grand Unified Models," Nucl. Phys. B 197, 533 (1982). S. Weinberg, "Supersymmetry at Ordinary Energies. 1. Masses and Conservation Laws," Phys. Rev. D 26 (1982) 287.

[20] H. Murayama and A. Pierce, "Not even decoupling can save minimal supersymmet- ric SU(5)," Phys. Rev. D 65, 055009 (2002) arXiv:hep-ph/0108104.

[21] B. Bajc, P. Fileviez Pérez and G. Senjanović, "Proton decay in minimal supersymmetric SU(5)," Phys. Rev. D 66, 075005 (2002) arXiv:hep-ph/0204311.

[22] B. Bajc, P. Fileviez Pérez and G. Senjanović, Minimal supersymmetric SU(5) theory and proton decay: Where do we stand?, arXiv:hep-ph/0210374.

[23] C. Bachas, C. Fabre and T. Yanagida, "Natural gauge-coupling unification at the string scale," Phys. Lett. B 370, 49 (1996) arXiv:hep-th/9510094. J. L. Chkareuli and I. G. Gogoladze, "Unification picture in minimal supersymmetric SU(5) model with string remnants," Phys. Rev. D 58 (1998) 055011 hep-ph/9803335.

[24] G. Dvali, "Black Holes and Large N Species Solution to the Hierarchy Problem," Fortsch. Phys. 58, 528 (2010) arXiv:0706.2050 [hep-th]].

[25] F. Takayama and M. Yamaguchi, "Gravitino dark matter without R-parity," Phys. Lett. B 485, 388 (2000) [hep-ph/0005214].

[26] B. Bajc, T. Enkhbat, D. K. Ghosh, G. Senjanović and Y. Zhang, "MSSM in view of PAMELA and Fermi-LAT," JHEP 1005, 048 (2010) arXiv:1002.3631 [hep-ph]].

[27] R. N. Mohapatra, "New Contributions To Neutrinoless Double-Beta Decay In Supersymmetric Theories," Phys. Rev. D 34, 3457 (1986); A. Font, L. E. Ibáñez and F. Quevedo, "Does Proton Stability Imply The Existence Of An Extra Z0?," Phys. Lett. B 228, 79 (1989); S. P. Martin, "Some simple criteria for gauged R-parity," Phys. Rev. D 46, 2769 (1992).

[28] C. S. Aulakh, B. Bajc, A. Melfo, A. Rašin and G. Senjanović, "SO(10) theory of R-parity and neutrino mass," Nucl. Phys. B 597, 89 (2001) hep-ph/0004031.

[29] C. S. Aulakh, A. Melfo, A. Rašin and G. Senjanović, "See-saw and supersymmetry or exact R-parity," Phys. Lett. B 459, 557 (1999) arXiv:hep-ph/9902409; 
[30] B. Bajc, A. Melfo, G. Senjanović and F. Vissani, "Yukawa sector in non-supersymmetric renormalizable SO(10)," Phys. Rev. D 73, 055001 (2006) arXiv:hep-ph/0510139.

[31] C.S. Aulakh, R.N. Mohapatra, "Implications Of Supersymmetric SO(10) Grand Unification," Phys. Rev. D 28, 217 (1983); T. E. Clark, T. K. Kuo and N. Nakagawa, "A SO(10) Supersymmetric Grand Unified Theory," Phys. Lett. B 115, 26 (1982). See also C. S. Aulakh, B. Bajc, A. Melfo, G. Senjanović and F. Vissani, "The Minimal supersymmetric grand unified theory," Phys. Lett. B 588 (2004) 196 hep-ph/0306242.

[32] K. S. Babu and R. N. Mohapatra, "Predictive neutrino spectrum in minimal $\mathrm{SO}(10)$ grand unification," Phys. Rev. Lett. 70, 2845 (1993) arXiv:hep-ph/9209215.

[33] G. Senjanović, "Proton decay and grand unification," AIP Conf. Proc. 1200 (2010) 131 arXiv:0912.5375 [hep-ph]].

[34] B. Bajc, G. Senjanović and F. Vissani, "b - tau unification and large atmospheric mixing: A case for non-canonical seesaw," Phys. Rev. Lett. 90, 051802 (2003) arXiv:hep-ph/0210207]; B. Bajc, G. Senjanović and F. Vissani, "Probing the nature of the seesaw in renormalizable $\mathrm{SO}(10)$," Phys. Rev. D 70, 093002 (2004) hep-ph/0402140.

[35] H. S. Goh, R. N. Mohapatra and S. -P. Ng, "Minimal SUSY SO(10), b tau unification and large neutrino mixings," Phys. Lett. B $\mathbf{5 7 0}$ (2003) 215 hep-ph/0303055].

[36] K. Abe et al. [T2K Collaboration], "Indication of Electron Neutrino Appearance from an Accelerator-produced Off-axis Muon Neutrino Beam," Phys. Rev. Lett. 107, 041801 (2011) arXiv:1106.2822 [hep-ex]].

[37] F. P. An et al. [DAYA-BAY Collaboration], "Observation of electron-antineutrino disappearance at Daya Bay," arXiv:1203.1669 [hepex].

[38] B. Bajc, I. Doršner and M. Nemevšek, "Minimal $\mathrm{SO}(10)$ splits supersymmetry," JHEP 0811, 007 (2008) arXiv:0809.1069 [hep-ph]].
[39] N. Arkani-Hamed and S. Dimopoulos, "Supersymmetric unification without low energy supersymmetry and signatures for finetuning at the LHC," JHEP 0506, 073 (2005) hep-th/0405159; G. F. Giudice and A. Romanino, "Split supersymmetry," Nucl. Phys. B 699, 65 (2004), [Erratum-ibid. B 706, 65 (2005)] hep-ph/0406088.

[40] B. Bajc and G. Senjanović, "Radiative seesaw: A Case for split supersymmetry," Phys. Lett. B 610, 80 (2005) hep-ph/0411193; B. Bajc and G. Senjanović, "Radiative seesaw and degenerate neutrinos," Phys. Rev. Lett. 95, 261804 (2005) hep-ph/0507169.

[41] E. Witten, "Neutrino Masses in the Minimal O(10) Theory," Phys. Lett. B 91, 81 (1980).

[42] K. S. Babu, J. C. Pati and F. Wilczek, "Fermion masses, neutrino oscillations, and proton decay in the light of Super-Kamiokande," Nucl. Phys. B 566, 33 (2000) hep-ph/9812538.

[43] G. Senjanović, "Neutrino mass: From LHC to grand unification," Riv. Nuovo Cim. 034, 1 (2011). 